\title{
Exponential or power distance-decay for commuting? An alternative specification
}

\author{
Jacob J de Vries $\uparrow$, Peter Nijkamp, Piet Rietveld \\ Department of Spatial Economics, Vrije Universiteit, De Boelelaan 1105, NL-1081HV Amsterdam, \\ The Netherlands; e-mail: jvries@feweb.vu.nl, pnijkamp@feweb.vu.nl, prietveld@feweb.vu.nl \\ Received 23 November 2006; in revised form 29 August 2007; published online 20 November 2008
}

\begin{abstract}
In this paper we investigate the functional form of distance decay for commuting flows between municipalities in Denmark. Our inference is based on a single equation that includes variables to capture the effect of spatial structure. Special attention is given to a proper estimation method: we estimate the distance-decay parameters by nonlinear weighted least-squares with balancing factors. It appears that neither an exponential nor a power distance-decay function fits the data well. Using a spline regression we find a cost elasticity of -4 for distances around $20 \mathrm{~km}$ and a much smaller value for shorter and longer distances. It appears that the logarithm of distance decay can be described adequately as a (downward) logistic function of log cost.
\end{abstract}

\section{Introduction}

Commuting has been the subject of increasing public attention and intensive research in recent decades (see Nijkamp and Rouwendal, 2004; Rouwendal and Nijkamp, 2004). The increasing role of commuting has far-reaching consequences for public policy concerning traffic, infrastructure, and spatial planning. Commuting flows connect labor and housing markets. Knowledge of the intensity and spatial range of this interaction is required to determine the accessibility of a location to centers of population and employment. Theoretical and applied research on commuting issues can help to clarify the relevant effects.

The analysis of the sizes of commuting flows between cities or regions, and their effects on housing and labor markets, is part of the much wider area of spatial interaction modeling (Batten and Boyce, 1986; Fotheringham and O'Kelly, 1989; Nijkamp and Reggiani, 1992). Spatial interaction models are applied not only to commuting but also to migration, international trade, shopping behavior, and other topics related to origin-destination flows. An essential element of these models is that interaction decreases with distance or travel cost. This is described by a distance-decay function. However, distance is not the only factor affecting the intensity of the interaction. Also important are the sizes (however measured) of origin and destination. And it is increasingly recognized that additionally the positions of origin and destination in the spatial system are relevant. The research in this paper is an application of spatial interaction modeling, and the methodological issues are not limited to commuting.

An important issue in spatial interaction modeling is the choice of the functional form of distance or travel cost. In the literature, the distance-decay function is usually assumed to be an exponential or a power function:

"In practice, the debate over the form of the cost function in spatial interaction models has evolved to a reasonably widespread consensus that the exponential function is more appropriate for analysing short distance interactions such as those that take place within an urban area. The power function, conversely, is generally held to be more appropriate for analysing longer distance interactions such as migration flows" (Fotheringham and O'Kelly, 1989, pages 12-13). 
Various other functional forms are suggested in the literature. Fik and Mulligan (1998) apply a specification with a Box-Cox (Box and Cox, 1964) transformation, which includes the exponential and power functions as special cases. In this paper we will propose a more flexible specification of the decay function. The functional form of distance decay should not only be based on theoretical considerations, but also empirically investigated (cf Taylor, 1975). The purpose of this paper is to investigate the functional form of distance decay for commuting in Denmark. For Denmark, a dataset containing all workers is available at a low level of spatial aggregation. We use data on commuting flows and travel costs between the 275 municipalities in Denmark in 1995.

Our inference is based on an equation that describes trip shares as being dependent on origin-specific and destination-specific effects and a function of travel cost. This equation occurs in several types of spatial interaction models, such as those proposed by Wilson (1971), Alonso's (1978) theory of movements, and competing-destinations models. Estimation of the distance-decay function is a first step in the estimation of a complete spatial interaction model. We will limit ourselves in the present paper to estimation of the decay function. The second step in the estimation of a spatial interaction model uses the estimated distance-decay function and balancing factors. Misspecification of the distance-decay function will affect the estimation of the other parameters in the model. Therefore it is important to be careful in the choice of functional form.

There are some econometric complications in the estimation of the trips equation of a spatial interaction model. First, the equation contains not only a distance-decay function but also unobserved balancing factors. Secondly, there is the issue how to handle the many zero flows. Thirdly, as the positive flows vary from one to thousands, heteroscedasticity can be considerable. Solutions for these problems have been proposed in the literature (summarized by Sen and Smith, 1995, pages 485-486). The econometric approach in this paper is based on those methods, but we introduce several new elements. We apply nonlinear weighted least-squares estimation (NLWLS) with balancing factors. The weights are based on a combination of Poisson error and lognormal specification error. As the weighting prohibits elimination of the balancing factors, we include them in the regression.

It appears that Danish commuting flows cannot be described by an exponential distance-decay function. A power specification gives better results, but the residual plot shows that the functional form is not correct. To investigate this, we applied a spline regression, allowing the elasticity of the decay function to differ in various ranges. The estimated elasticities (in absolute value) increase for small distances $(<15 \mathrm{~km})$, reach a maximum of 4 for intermediate distances $(15-30 \mathrm{~km})$, and decrease gradually for larger distances. This ad hoc specification can be approximated using a logistic function. In our final specification, the logarithm of the decay function is a downward logistic function of log cost.

The paper is structured as follows. Section 2 discusses spatial interaction modeling and distance-decay functions. Section 3 describes the data, and section 4 the estimation method. In section 5 we estimate various distance-decay functions. In section 6 we discuss the results and conclude. Several derivations are placed in appendices A and $\mathrm{B}$.

\section{Theory}

In this section we provide the theoretical background for the empirical research presented later on in this paper. We introduce notation and our model framework, and demonstrate how that fits into several types of spatial interaction models. Also, we review specifications of the distance-decay function found in the literature. 
For a general introduction to spatial interaction models, we refer to Fotheringham and O'Kelly (1989).

Our point of departure is the following model framework:

$$
\begin{aligned}
T_{i j} & =A_{i} B_{j} O_{i} D_{j} Q^{-1} F_{i j}, \\
O_{i} & =\sum_{j} T_{i j}, \\
D_{j} & =\sum_{i} T_{i j}, \\
Q & =\sum_{i} O_{i}=\sum_{j} D_{j}=\sum_{i, j} T_{i j},
\end{aligned}
$$

where $T_{i j}$ ('trips') is the number of people living in municipality $i$ and working in municipality $j ; A_{i}$ and $B_{j}$ are unobserved balancing factors, which make it possible to satisfy equations (2) and (3); $O_{i}$ is the total number of working people living in (origin) municipality $i$; $D_{j}$ is the total number of workers in (destination) municipality $j$; $Q$ is the overall total number of workers; and $F_{i j}$ is the distance-decay function, a decreasing function of the travel cost from municipality $i$ to municipality $j$. We included $Q$ in equation (1) to capture the scale of the data, so that the balancing factors and the decay function are dimensionless.

Several types of spatial interaction models can be represented in a form that contains these equations (Batten and Boyce, 1986; de Vries et al, 2001). In the genuine doubly constrained model, $O_{i}$ and $D_{j}$ are assumed to be exogenous. In the unconstrained gravity model (Carey, 1858; Stewart, 1941) $O_{i}$ is proportional to accessibility to jobs $\left(A_{i}^{-1}\right)$ and the size $V_{i}$ of the origin, and $D_{j}$ is proportional to accessibility to the labor force $\left(B_{j}^{-1}\right)$ and size $W_{j}$ of the destination. Singly constrained models (Wilson, 1971) are a combination of these. Alonso's theory of movements (Alonso, 1978; Bikker, 1987; Bikker and De Vos, 1992; Hamerslag, 1980) results by adding the equations:

$$
\begin{aligned}
& O_{i}=V_{i} A_{i}^{-\alpha}, \\
& D_{j}=W_{j} B_{j}^{-\beta}
\end{aligned}
$$

(de Vries et al, 2001). According to Ledent (1980), Poot (1986), and de Vries et al (2002) estimation of Alonso's theory of movements can proceed in two stages, where the first stage consists of estimation of the distance-decay function and the systemic variables $A_{i}$ and $B_{j}$. In this paper we perform this first stage. (The true challenge is the estimation of $\alpha$ and $\beta$ in the second stage.) A similar two-stage approach is possible for the competing-destinations model. In a production-constrained competingdestinations model (Pellegrini and Fotheringham, 1999), $O_{i}$ is exogenous and the fraction from origin $i$ choosing destination $j$ is modeled as

$$
\frac{T_{i j}}{O_{i}}=\frac{W_{j} F_{i j} L_{j}^{\vartheta}}{\sum_{k} W_{k} F_{i k} L_{k}^{\vartheta}},
$$

where $L_{j}$ is the competing-destinations indicator. If we define $A_{i}^{-1}=Q^{-1} \sum_{j} W_{j} F_{i j} L_{j}^{\vartheta}$, and $B_{j}^{-1}=D_{j} W_{j}^{-1} L_{j}^{-\vartheta}$ substitution shows that equation (7) is equivalent to equation (1). So the analysis in this paper can function in the context of several types of spatial interaction model. 
In this paper we do not use a complete model, but only estimate the distance-decay function, based on equations (1)-(4). These equations contain almost all information on the distance-decay function. Involving the other model equations [such as (5) and (6) or (7)] into the estimation procedure would slightly improve efficiency, but is much more complicated. Equations (2)-(4) are definitions of $O_{i}, D_{j}$, and $Q$. We can rewrite equation (1) as

$$
\frac{T_{i j}}{Q} /\left(\frac{O_{i}}{Q} \frac{D_{j}}{Q}\right)=A_{i} B_{j} F_{i j}
$$

This formulation can be seen as a probability, conditional on the border totals (Batty and Sikdar, 1982; Mazurkiewicz, 1982). If there is no effect of travel cost $\left(F_{i j}=1, \forall i, \forall j\right)$ and no effect of spatial structure $\left(A_{i}=1, \forall i\right.$, and $\left.B_{j}=1, \forall j\right)$, the right-hand side of equation (8) equals 1 , and the result can be called 'random commuting' (Thorsen et al, 1999). Our task in this paper is to decompose the observed left-hand side of equation (8) into the spatial-structure effects $A_{i}$ for origins and $B_{j}$ for destinations, and the spatial-separation effect $F_{i j}$ as a decay function of travel cost.

In the literature, it is generally agreed that an exponential specification,

$$
F_{i j}=\gamma_{0} \exp \left(\gamma_{1} G_{i j}\right)
$$

is in accordance with a utility framework (Cochrane, 1975). However, in applications often a power specification,

$$
F_{i j}=\gamma_{0} G_{i j}^{\gamma_{1}},
$$

appears to have a better fit. Choukroun (1975) provides a possible theoretical justification for a power specification. Deviations from an exponential form towards a power form can be caused by heterogeneity of user preferences, use of various travel modes, and lower trip frequency for long-distance commuters. The resulting functional form depends on the mathematical assumptions concerning the heterogeneity. Other types of decay functions are not excluded in advance. Taylor (1975) investigated not only the exponential and power (Pareto) specification, but also normal, lognormal, and squareroot exponential. Box - Cox (1964) transformations (Fik and Mulligan, 1998) are more general, and include the exponential and the power specification as special cases.

Several researchers found that the sensitivity of commuters with respect to travel cost (or time or distance) is stronger for intermediate distances than for short and long distances (Johansson et al, 2002; 2003). To model this, an S-shaped decay function is required. Hilbers and Verroen (1993) and Geurs and Ritsema van Eck (2003) apply a log-logistic decay function:

$$
F_{i j}=\frac{\gamma_{0}}{1+\exp \left(\gamma_{1}+\gamma_{2} \ln G_{i j}\right)} .
$$

Thorsen et al (1999) provide a theoretical justification for such an S-shaped curve, based on the idea that short distances give random commuting flows, whereas long distances are governed by a minimum cost principle.

Taylor (1975) proposes to analyze the relationship between interaction and distance by regressing the logarithm of interaction intensity on various transformations of distance and choosing the best fitting specification. We will follow a similar approach. We try to answer the question concerning the functional form of the distance-decay function from an empirical starting point. So we specify $F_{i j}$ very generally as

$$
\ln F_{i j}=\mathrm{f}\left(G_{i j}\right)+u_{i j} .
$$


Note that $\mathrm{f}$ is not the distance-decay function itself, but its logarithm. For most functional forms this is a convenient representation: for example, if the decay function is exponential, $\mathrm{f}$ is linear.

Our specification of the distance-decay function is rather general, but it still implies some constraints. We do not allow origin-specific or destination-specific parameters in the decay function. The fit of the model can be improved by having each origin its own distance-decay parameter, but it has been demonstrated (Fotheringham, 1984; Tiefelsdorf, 2003) that this can lead to biased estimates, as the estimated distance-decay parameter picks up the effect of spatial structure. Omission of the spatial-structure variables can result in biased estimates of the decay function, but the reverse is also true. If the distance-decay function is misspecified, the systemic variables (opportunities and competition indicators) in Alonso's (1978) theory of movements, or the measure of centrality in Fotheringham's (1983) competing-destinations model, will not be computed with the correct value (Tiefelsdorf, 2003). As a consequence, estimates of the parameters associated with these variables will be biased, and wrong predictions can result. "Interaction modeling and accessibility studies must take extra care in specifying the global distance decay relationship to avoid introducing a spatial structure bias" (Tiefelsdorf, 2003, page 48).

Our inference will be based on equation (8). If we take logs and substitute equation (12) for $\ln F_{i j}$, we obtain:

$$
\ln T_{i j}-\ln O_{i}-\ln D_{j}+\ln Q=\ln A_{i}+\ln B_{j}+\mathrm{f}\left(G_{i j}\right)+u_{i j} .
$$

The construction of travel cost $G_{i j}$ will be discussed in the next section. Assumptions on the disturbance term $u_{i j}$ will be stated in section 4 . The form of the function $\mathrm{f}$ will be chosen in section 5 .

\section{Data}

The estimation of the distance-decay function will be based on data on commuting between municipalities in Denmark in 1995. Figure 1 shows the counties of Denmark. Bridges and ferries provide the major connections between the islands. In 1995 the fixed (road and rail) link over the Great Belt was not yet finished.

The data for $T_{i j}$ are derived from the population census: a $275 \times 275$ table of the active population in Denmark in 1995 by municipality of residence and municipality of work. Of the over 75000 data points on municipality-to-municipality commuting flows, $64 \%$ are 0 . As will be discussed in the next section, the zero flows are included in the analysis, but the weight they get is small if the expected flow is close to zero. Also the positive flows are usually small: $13 \%$ of the observations are equal to $1,14 \%$ lie between 2 and 10, and only $9 \%$ are larger than 10 . The largest flows are of course those within large cities such as Copenhagen (138202) and Aarhus (121480). The largest flows between different municipalities are those between Copenhagen and nearby municipalities: 20846 commuters from Frederiksberg to Copenhagen and 12808 the other way around, and 12949 commuters from Gentofte to Copenhagen. $O_{i}, D_{j}$, and $Q$ are computed from $T_{i j}$ using equations (2), (3), and (4).

The available cost data consist of a $275 \times 275$ table of the financial costs of commuting trips between municipalities by car, in Danish crowns (DKK). These costs have been constructed by Andersen (1999a) as the land distance times a kilometer price (set to $1 \mathrm{DKK} / \mathrm{km}, 1 \mathrm{DKK}$ is approximately 13 eurocents), plus the rate of a possible ferry (for a car and driver), minus the tax deduction for commuting cost. The distance within a municipality is computed as the average distance to the center from points within a circle with the same area as the municipality (Andersen, 1999b, page 26), so $\frac{2}{3} r$ or $\frac{2}{3}(\text { area } / \pi)^{1 / 2}$. The fact that the kilometer rate is set to the round number of 


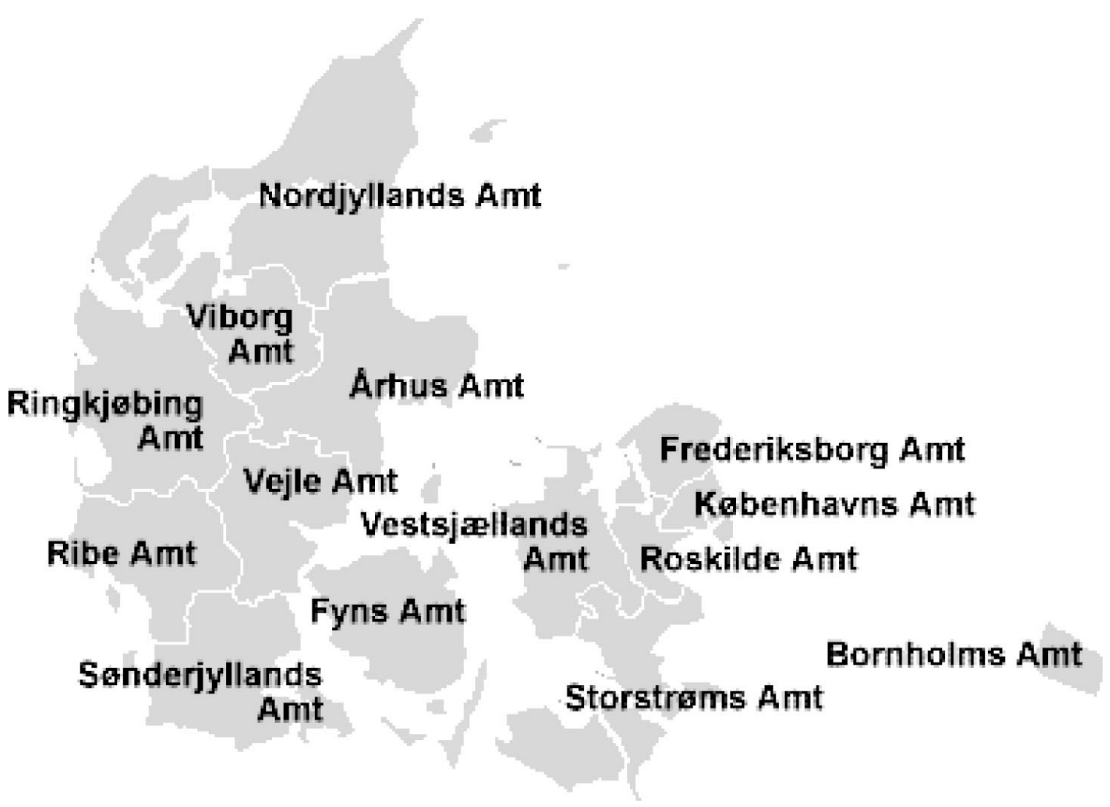

Figure 1. Map of Denmark, with division into counties. The Great Belt is the strait between Vestsjællands Amt and Fyns Amt.

$1 \mathrm{DKK}$ implies that the cost numerically equals the distance, as long as no ferries are involved and we ignore the tax deduction. This will be useful for the interpretation of the estimated distance-decay functions. (If the actual kilometer price were to deviate from this, the interpretation in kilometers would still hold, but a correction to ferry rates would be required.) Of the working population 59\% works in the municipality of residence; $5 \%$ has a commuting cost of over 30 DKK (approximately equivalent to $30 \mathrm{~km}$ ); and $1 \%$ has a commuting cost of over $200 \mathrm{DKK}$, which usually includes a ferry passage.

As these cost data are artificially constructed, they might deviate from the generalized costs as perceived by the commuters. Generalized costs include not only monetary costs, but also time costs and other aspects, such as comfort and reliability. A first source of error has to do with ferry connections. Ferry rates are included in the cost measure, but time costs are not. Usually, distance, cost, and travel time are highly correlated, but in the case of ferries the ratio might differ from that on the roads. Further, the cost is based on the rate for bringing a car on the ferry, while some commuters will use the ferry as foot passengers or by train (in both cases at lower cost). Therefore we include dummy variables for travel over the Great Belt and from or to the islands Bornholm and Laeso. In and around Copenhagen congestion might increase generalized travel costs, and therefore we include dummy variables for Copenhagen and rings around it. Exact definitions of the dummy variables are given in table 1. The dummy ChriVej serves to compensate an obvious measurement error in the distance. Both sides of the Isefjord (the bay in the north of Zealand) are on the same island, and the data do not include the ferry cost here, so we include a dummy variable to correct for this.

The cost data $C_{i j}$ are combined with the dummy variables in order to obtain the corrected travel cost $G_{i j}$ :

$$
\begin{aligned}
G_{i j}= & C_{i j}+\delta_{1} \text { GreatBelt }_{i j}+\delta_{2} \text { Bornholm }_{i j}+\delta_{3} \text { Laeso }_{i j} \\
& +\left(\delta_{4} \text { CopCity }_{i j}+\delta_{5} \text { CopCounty }_{i j}+\delta_{6} \text { CopRegion }_{i j}+\delta_{7} \text { ChriVej }_{i j}\right) C_{i j} \\
& +\delta_{8} \text { Isefjord }_{i j} / C_{i j} .
\end{aligned}
$$


Table 1. Definitions of dummy variables.

Dummy variable ${ }^{a}$ is 1 for and only for ( 0 else)

GreatBelt trips from Københavns Amt, Frederiksborg Amt, Roskilde Amt, Vestsjællands Amt, Storstrøms Amt, Bornholms Amt, to Flyns Amt, Søderjyllands Amt, Ribe Amt, Vejle Amt, Ringkjøbing Amt, Ârhus Amt, Viborg Amt, Nordjyllands Amt, or the other way around.

Bornholm trips from or to Bornholms Amt (but not within Bornholms Amt).

Laeso trips from or to the municipality Laeso (but not within Laeso).

CopCity trips within or between the municipalities Copenhagen and Frederiksberg.

CopCounty trips within Københavns Amt, excluding those for which CopCity applies.

CopRegion trips within or between Københavns Amt, Frederiksborg Amt and

Roskilde Amt, excluding those for which CopCity or CopCounty applies.

Chrivej

Isefjord trips between the municipalities Christiansfeld and Vejen.

trips from Frederiksborg Amt to Vestsjællands Amt, or the other way around.

a In the definition of dummy variables Københavns Amt (county Copenhagen) is taken to include the municipalities Copenhagen and Frederiksberg, which are formally not part of the country Copenhagen but enjoy their own county status.

The correction for the use of a ferry is a fixed amount, while the correction for congestion is proportional to the cost. $G_{i j}$ is a model approximation for the generalized costs, containing parameters to be estimated simultaneously with the decay function. For estimation, equation (14) is substituted in equation (13), resulting in a nonlinear equation (except for the exponential decay function).

\section{Econometrics}

The estimation method that we use in this paper combines known elements from the literature with a few innovations. We estimate the parameters of the decay function by NLWLS, including the balancing factors as fixed origin and destination effects. The weights are based on a combination of specification error and Poisson error. For the treatment of small and zero flows, we use a method developed by Sen and Soot (1981). Our estimation method is similar to "Procedure 1" of Sen and Smith (1995, pages $485-486$ ), but differs in three respects. Firstly, it is nonlinear, to allow for more complicated decay functions. Secondly, the weights differ, as we think that specification error cannot be ignored. Thirdly, as the use of weights prohibits application of the decomposition method we include origin and destination dummies in the least-squares procedure. In the remainder of this section we describe the estimation method in more detail.

The estimation is based on equation (13):

$$
\ln T_{i j}-\ln O_{i}-\ln D_{j}+\ln Q=\ln A_{i}+\ln B_{j}+\mathrm{f}\left(G_{i j}\right)+u_{i j},
$$

where $G_{i j}$ is given by equation (14). The left-hand side is observed. This equation contains the endogenous variables $A_{i}$ and $B_{j}$, but there is no endogeneity problem, as these variables do not have an associated parameter. $A_{i}$ and $B_{j}$ are latent variables and will be estimated as origin-specific and destination-specific dummy variables. The information on $A_{i}$ and $B_{j}$ which is present in other equations of the model is ignored here. The loss of efficiency is limited, as equation (13) represents $275 \times 275$ degrees of freedom, while the rest of the model contains only $2 \times 275$ degrees of freedom and includes additional parameters. 
The logarithmic transformation of the dependent variable in equation (13) causes a bias, as the expectation of the logarithm is not equal to the logarithm of the expectation (Sen and Soot, 1981, page 167). Moreover, for the many zero flows the logarithm does not exist. Therefore we apply the suggestion of Sen and Soot (1981, page 167) to use $\ln \left(T_{i j}+\frac{1}{2}\right)$ instead of $\ln \left(T_{i j}\right)$ in the dependent variable. Under the assumption that the trips have a Poisson distribution (so var $T_{i j}=E T_{i j}$ ), a Taylor approximation around $E T_{i j}$ shows (Sen and Smith, 1995, page 480) that:

$$
E \ln \left(T_{i j}+\frac{1}{2}\right) \approx \ln E T_{i j} .
$$

Proof

$$
\begin{aligned}
E \ln \left(T_{i j}+\frac{1}{2}\right) & \approx \mathrm{E}\left[\ln E T_{i j}+\frac{1}{E T_{i j}}\left(T_{i j}+\frac{1}{2}-E T_{i j}\right)-\frac{1}{2} \frac{1}{\left(E T_{i j}\right)^{2}}\left(T_{i j}+\frac{1}{2}-E T_{i j}\right)^{2}\right] \\
& =\ln E T_{i j}+\frac{1}{2} \frac{1}{E T_{i j}}-\frac{E\left[\left(T_{i j}-E T_{i j}\right)^{2}+\left(T_{i j}-E T_{i j}\right)+\frac{1}{4}\right]}{2\left(E T_{i j}\right)^{2}} \\
& =\ln E T_{i j}+\frac{1}{2 E T_{i j}}-\frac{\operatorname{var} T_{i j}}{2\left(E T_{i j}\right)^{2}}-\frac{1}{8\left(E T_{i j}\right)^{2}}=\ln E T_{i j}-\frac{1}{8\left(E T_{i j}\right)^{2}} .
\end{aligned}
$$

If $E T_{i j}$ is not too small, the latter term can be neglected.

So the use of $\ln \left(T_{i j}+\frac{1}{2}\right)$ in the dependent variable strongly reduces the bias (Sen and Soot, 1981; Sen and Smith, 1995, page 480). It also provides a solution to the problem of zero flows.

Sen and Soot (1981, page 168) and Sen and Smith (1995, page 484) suggest applying WLS, as heteroscedasticity may be expected. They assume that the flows have a Poisson distribution. In that case:

$$
\operatorname{var} \ln \left(T_{i j}+\frac{1}{2}\right) \approx \frac{1}{E T_{i j}},
$$

and weights can be derived from that. The Poisson distribution is suitable to account for the dispersion caused by the individual choices of the commuters around the modeled expectation. However, there is another source of error: the specification error. There are factors affecting the commuting pattern that are not included in the model. If this is ignored, large flows will get much too heavy a weight (de Vries et al, 2002). An alternative approach is to assume that $u_{i j}$ has constant variance $\sigma_{u}^{2}$. This will work well to treat specification error, but ignores the fact that small flows have a relatively larger standard deviation. Therefore, we combine both variances, ${ }^{(1)}$ and assume

$$
\operatorname{var}\left(u_{i j}\right)=\sigma_{u}^{2}+\frac{1}{E T_{i j}}=\sigma_{u}^{2}\left(1+\frac{1}{\sigma_{u}^{2} E T_{i j}}\right) .
$$

Overdispersion can also be modeled by a negative binomial distribution. After taking logs the same variance specification is the result. We estimate $\sigma_{u}^{2}$ in the regression, and weight the squared residuals by

$$
w_{i j}^{2}=\left(1+\frac{1}{\sigma_{u}^{2} E T_{i j}}\right)^{-1} .
$$

(1) The introduction of specification error has no consequences for the procedure of adding $\frac{1}{2}$ to each observation before taking logs. The data-generating process can be considered to consist of two steps. A value drawn from a lognormal distribution is used as the expected value in a Poisson distribution. Taking logs of a Poisson variable requires correction by adding $\frac{1}{2}$. Taking logs of a lognormal variable results in a normally distributed variable. 
This implies that zero flows get a very small weight if the expected flow is close to zero, while the weight approaches 1 for large expected flows. The weights depend on both $\sigma_{u}^{2}$ and $E T_{i j}$, which have to be estimated, so we iterate between the WLS procedure and the calculation of the weights.

For most choices of $f$, the equation resulting from substitution of equation (14) into equation (13) is nonlinear. So we apply nonlinear least squares (Stoer and Bulirsch, 1980, pages 209-210). This is an iterative procedure. A regression based on a linear approximation around the most recent parameter estimates results in updates for these estimates. The iteration for the weights can be integrated in the iteration of the nonlinear least-squares procedure.

The unobserved balancing factors $A_{i}$ and $B_{j}$ in equation (13) need to be estimated. Therefore we include dummy variables for all origins and destinations. (We impose the restrictions that the weighted sum of origin dummy parameters, as well as the weighted sum of destination dummy parameters, equals zero.) However, with 550 dummy variables, a standard regression procedure will take a considerable amount of computer time and memory (Sen and Smith, 1995, page 476). Therefore, Cesario (1974, page 252) suggested decomposing equation (13) into the overall average, and $i$-component, a $j$-component, and the remainder. The last component is the deviation from the average over $i$ and the average over $j$, so the balancing factors cancel. The parameters of the distance-decay function can then be estimated by regression (Cesario, 1974, page 252; Sen and Smith, 1995, page 477). However, in case of WLS this method is no longer equivalent to the complete regression (see appendix A). The use of weighted averages in the decomposition (de Vries et al, 2002) does not fully solve this problem. Therefore we chose a different approach. Generally, the parameter estimates for WLS are given by the following formula:

$$
\left(\mathbf{X}^{\mathrm{T}} \mathbf{W} \mathbf{X}\right)^{-1} \mathbf{X}^{\mathrm{T}} \mathbf{W} \boldsymbol{y}
$$

We directly construct $\mathbf{X}^{\mathrm{T}} \mathbf{W} \mathbf{X}$ and $\mathbf{X}^{\mathrm{T}} \mathbf{W} \boldsymbol{y}$ in our program, invert $\mathbf{X}^{\mathrm{T}} \mathbf{W} \mathbf{X}$ numerically, and compute the parameter estimates using equation (19). In this way we avoid the storage $(300 \mathrm{MB})$ of the $\mathbf{X}$ matrix. Inverting a $560 \times 560$ matrix appears to be no problem (it takes $0.7 \mathrm{~s}$ ). Appendix A describes this procedure in more detail.

To summarize, we estimate

$$
\ln \left(T_{i j}+\frac{1}{2}\right)-\ln O_{i}-\ln D_{j}+\ln Q=\ln A_{i}+\ln B_{j}+\mathrm{f}\left(G_{i j}\right)+u_{i j},
$$

where $G_{i j}$ is given by equation (14), by NLWLS, using weights given in equation (18). The computations were done using the object-oriented matrix programming language Ox (Doornik, 1998). (2) This procedure results in estimates for the parameters of the distance-decay function, the dummy parameters in $G_{i j}$, and balancing factors for all origins and destinations. In the next section we will apply this method to various specifications of the decay function.

\section{Inference}

In this section we analyze the relation between trips, origins, destinations, and travel cost. We want to decompose the observations on the left-hand side of equation (20) into balancing factors, a function of cost and geographical dummies, and a disturbance term. This is similar to the approach of Taylor (1975), but origin-specific and destination-specific variables are included in the equation to capture the effect of spatial structure and avoid bias in the estimation of the distance-decay function. 
Figure 2 shows the left-hand side of equation (20) against the log of observed cost. We estimate four specifications for the distance-decay function. Table 2 lists the parameter estimates. $^{(3)}$ Figure 3 shows the residuals as a function of log cost, to judge the correctness of the functional form.

As a starting point we estimate the two most commonly applied decay functions: the exponential and the power:

$$
\begin{aligned}
& \ln \left(T_{i j}+\frac{1}{2}\right)-\ln O_{i}-\ln D_{j}+\ln Q=\gamma_{0}+\ln A_{i}+\ln B_{j}+\gamma_{1} G_{i j}+u_{i j}, \\
& \ln \left(T_{i j}+\frac{1}{2}\right)-\ln O_{i}-\ln D_{j}+\ln Q=\gamma_{0}+\ln A_{i}+\ln B_{j}+\gamma_{1} \ln G_{i j}+u_{i j} .
\end{aligned}
$$

Note that travel cost $G_{i j}$ is a function that includes several dummies, see equation (14). The power function performs much better than the exponential decay function, but is still not the correct functional form. To investigate the functional form, we perform a spline regression based on the power decay function. The spline function is on intervals linear in $\ln G_{i j}$, is continuous, but changes in slope at various points. We set the knots at travel cost of $8,15,30,50,100$, and 150 . (These values can roughly be interpreted as distances in kilometers.)

$$
\begin{aligned}
\ln \left(T_{i j}+\frac{1}{2}\right)-\ln O_{i}-\ln D_{j}+\ln Q= & \gamma_{0}+\ln A_{i}+\ln B_{j}+\gamma_{1} \ln \min \left(G_{i j}, 8\right) \\
& +\gamma_{2} \ln \max \left[8, \min \left(G_{i j}, 15\right)\right] \\
& +\gamma_{3} \ln \max \left[15, \min \left(G_{i j}, 30\right)\right] \\
& +\gamma_{4} \ln \max \left[30, \min \left(G_{i j}, 50\right)\right] \\
& +\gamma_{5} \ln \max \left[50, \min \left(G_{i j}, 100\right)\right] \\
& +\gamma_{6} \ln \max \left[100, \min \left(G_{i j}, 150\right)\right] \\
& +\gamma_{7} \ln \max \left(150, G_{i j}\right)+u_{i j} .
\end{aligned}
$$

The estimated decay function has a clear pattern. For cost $<8$, it is flatter than the power function. Between 15 and 30, it is very steep, with an elasticity that is in absolute value greater than 4 . For cost $>30$, the function becomes gradually flatter: when the cost doubles, the slope approximately halves. This regularity for larger costs suggests to find a function with this form: for example, the inverse exponential

$$
\mathrm{f}\left(G_{i j}\right)=\gamma_{0}+\frac{\gamma_{1}}{G_{i j}} .
$$

(In appendix B we show that this function has the desired property.) But then the decay function would still consist of several parts: this inverse exponential function for high cost, the standard exponential for low cost, and maybe a power function for the intermediate part.

Therefore we looked for an S-shaped function, with the correct behavior in the right tail. Most functional forms suggested in the literature have a curvature in only one direction. This holds for Taylor's (1975) normal, lognormal, and square-root

(3) The standard errors of the parameter estimates are underestimated, as the assumption of independent, identically distributed disturbances is violated. Differences in quality of the transportation network will affect multiple flows, as a given road generally forms part of the route for several origin - destination combinations. 


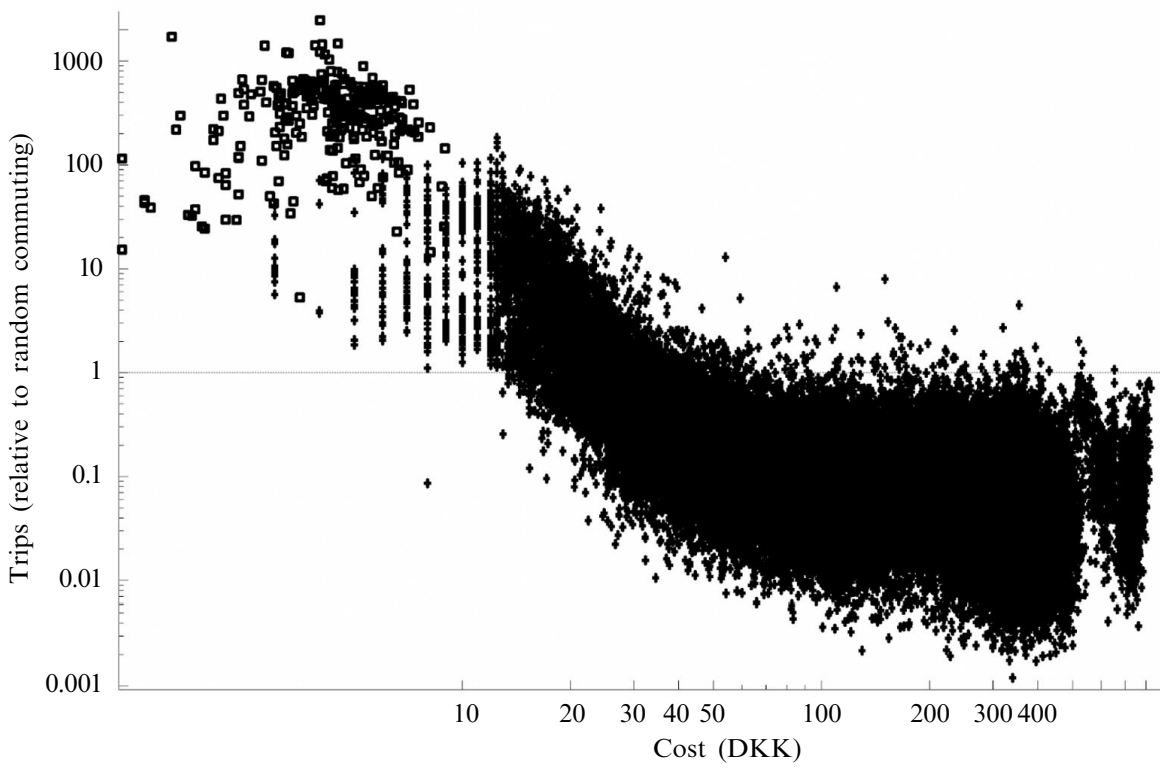

Figure 2. The logarithm of the commuting flow (relative to random commuting) as a function of the logarithm of travel cost (flows within municipalities represented by squares). If the decay function were a power function, the points would lie around a straight line.
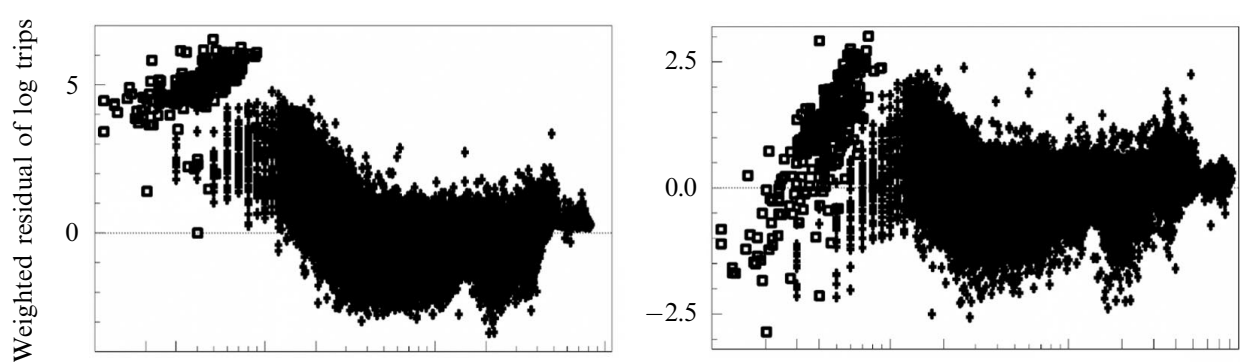

(a)

(b)
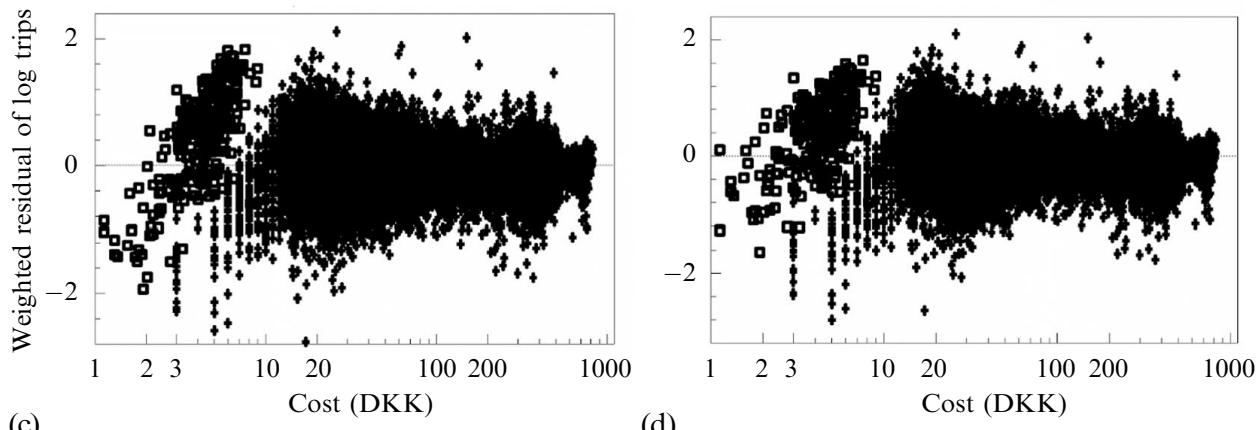

(d)

Figure 3. Weighted residuals of various decay functions (as function of the logarithm of travel cost): (a) exponential, (b) power, (c) power spline, (d) logistic based. Residuals for flows within municipalities are represented by squares. 
Table 2. Estimation results for various decay functions.

\begin{tabular}{|c|c|c|c|c|}
\hline & Exponential & Power & Power spline & Logistic based \\
\hline$R^{2}$ & 0.524 & 0.830 & 0.898 & 0.896 \\
\hline$\sigma$ & 0.753 & 0.336 & 0.231 & 0.234 \\
\hline Constant & $\begin{array}{l}-0.179 * * * * \\
(0.010)\end{array}$ & $\begin{array}{l}7.997 * * * * \\
(0.018)\end{array}$ & $\begin{array}{l}45.500 * * * * \\
(0.121)\end{array}$ & $\begin{array}{l}-3.745^{* * * *} \\
(0.009)\end{array}$ \\
\hline Cost & $\begin{array}{l}-0.0179 * * * * \\
(0.0001)\end{array}$ & & & \\
\hline $\operatorname{Ln}(\cos t)$ & & $\begin{array}{l}-2.350^{* * * * *} \\
(0.005)\end{array}$ & $\begin{array}{l}-1.870^{* * * * *} \\
(0.029)\end{array}$ & \\
\hline$>8$ & & & $\begin{array}{l}-2.439 * * * * \\
(0.031)\end{array}$ & \\
\hline$>15$ & & & $\begin{array}{l}-4.117 * * * * \\
(0.018)\end{array}$ & \\
\hline$>30$ & & & $\begin{array}{l}-2.536^{* * * * *} \\
(0.026)\end{array}$ & \\
\hline$>50$ & & & $\begin{array}{l}-1.699 * * * * \\
(0.024)\end{array}$ & \\
\hline$>100$ & & & $\begin{array}{l}-0.761 * * * * \\
(0.042)\end{array}$ & \\
\hline$>150$ & & & $\begin{array}{l}-0.543 * * * * \\
(0.022)\end{array}$ & \\
\hline $\begin{array}{l}\text { Logistic function } \\
{[\ln (\cos t)]}\end{array}$ & & & & $\begin{array}{l}9.806^{* * * * *} \\
(0.030)\end{array}$ \\
\hline Bending point & & & & $\begin{array}{l}19.845^{* * * * *} \\
(0.075)\end{array}$ \\
\hline Steepness & & & & $\begin{array}{l}1.509 * * * * \\
(0.007)\end{array}$ \\
\hline GreatBelt & $\begin{array}{c}-147.02 * * * * \\
(0.88)\end{array}$ & $\begin{array}{c}-128.50 * * * * \\
(0.54)\end{array}$ & $\begin{array}{c}-95.30 * * * * \\
(2.04)\end{array}$ & $\begin{array}{c}-96.27 * * * * \\
(2.11)\end{array}$ \\
\hline Bornholm & $\begin{array}{c}-138.71 * * * * \\
(5.00)\end{array}$ & $\begin{array}{c}-231.77 * * * * \\
(3.18)\end{array}$ & $\begin{array}{c}-234.44 * * * * \\
(9.07)\end{array}$ & $\begin{array}{c}-255.06^{* * * *} \\
(5.59)\end{array}$ \\
\hline Laeso & $\begin{array}{l}96.96^{* * * * *} \\
(21.78)\end{array}$ & $\begin{array}{c}-0.47 \\
(8.31)\end{array}$ & $\begin{array}{l}-43.23 * * * * \\
(3.29)\end{array}$ & $\begin{array}{l}-44.65^{* * * * *} \\
(2.71)\end{array}$ \\
\hline CopCity & $\begin{array}{l}-1.000 \\
\text { (lower bound) }\end{array}$ & $\begin{array}{l}3.606^{* * * * *} \\
(0.337)\end{array}$ & $\begin{array}{l}3.156^{* * * * *} \\
(0.149)\end{array}$ & $\begin{array}{l}2.995 * * * * \\
(0.152)\end{array}$ \\
\hline CopCounty & $\begin{array}{c}-1.000^{* * * *} \\
\text { (lower bound) }\end{array}$ & $\begin{array}{l}0.575^{* * * * *} \\
(0.019)\end{array}$ & $\begin{array}{l}0.428 * * * * \\
(0.008)\end{array}$ & $\begin{array}{l}0.441^{* * * * *} \\
(0.009)\end{array}$ \\
\hline CopRegion & $\begin{array}{c}-0.180^{* *} \\
(0.079)\end{array}$ & $\begin{array}{l}0.371^{* * * * *} \\
(0.014)\end{array}$ & $\begin{array}{l}0.094^{* * * * *} \\
(0.006)\end{array}$ & $\begin{array}{l}0.099^{* * * * *} \\
(0.006)\end{array}$ \\
\hline ChriVej & $\begin{array}{l}-1.000 \\
\text { (lower bound) }\end{array}$ & $\begin{array}{l}1.687^{* * * * *} \\
(0.329)\end{array}$ & $\begin{array}{l}1.787 * * * * \\
(0.154)\end{array}$ & $\begin{array}{l}1.786^{* * * * *} \\
(0.175)\end{array}$ \\
\hline Isefjord & $\begin{array}{l}3176.0 * * * * \\
(115.8)\end{array}$ & $\begin{array}{l}895.4 * * * * \\
(38.6)\end{array}$ & $\begin{array}{l}325.2 * * * * \\
(19.9)\end{array}$ & $\begin{array}{l}313.8^{* * * * *} \\
(17.0)\end{array}$ \\
\hline
\end{tabular}

Note. Standard errors are given in parenthesis.

$* 10 \%$ significant; $* * 5 \%$ significant, *** $1 \%$ significant, **** $0.1 \%$ significant.

exponential specification. Box-Cox (1964) transformations (Fik and Mulligan, 1998) allow curvature in both directions (depending on the sign), but not in combination (Tiefelsdorf, 2003). However, the power spline decay function in equation (23) can be approximated using a (downward) logistic function of $\ln G_{i j}$ :

$$
\mathrm{f}\left(G_{i j}\right)=\gamma_{0}+\frac{\gamma_{1}}{1+\gamma_{2}^{-\gamma_{3}} \exp \left(\gamma_{3} \ln G_{i j}\right)}=\gamma_{0}+\gamma_{1} /\left[1+\left(\frac{G_{i j}}{\gamma_{2}}\right)^{\gamma_{3}}\right] .
$$


This is demonstrated in appendix B. A logistic function has the properties that we noted. It started rather flat, then becomes steeper, and then gradually flatter again. The parameter $\gamma_{2}$ is the location of the bending point (in the same units as travel cost). The parameter $\gamma_{3}$ governs the steepness of the logistic function. Note that on the lefthand side of equation (25) is the logarithm of the decay function, while the log-logistic decay function (11) of Hilbers and Verroen (1993) has the decay function itself on the left-hand side.

So finally we estimated the logistic-based decay function

$$
\ln \left(T_{i j}+\frac{1}{2}\right)-\ln O_{i}-\ln D_{j}+\ln Q=\gamma_{0}+\ln A_{i}+\ln B_{j}+\gamma_{1} /\left[1+\left(\frac{G_{i j}}{\gamma_{2}}\right)^{\gamma_{3}}\right]+u_{i j} .
$$

The bending point of the logistic-based distance-decay function is estimated at $20 \mathrm{DKK}$ (approximately $20 \mathrm{~km}$ ), and there the elasticity reaches the value of $-\frac{1}{4} \gamma_{1} \gamma_{3}=-3.70$. The estimated parameters for the ferry dummies are all negative, reflecting that the cost is overestimated in the data. In and around Copenhagen, travel cost per kilometer is up to $300 \%$ higher. Figure 3(d) shows that the problems with the functional form have been solved, although there is still some heteroscedasticity left. Outliers for flows between nearby municipalities might be caused by specific spatial configuration of labor force and jobs around Copenhagen.

In this section we estimated four specifications of the decay function. We started with the two standard forms: the exponential and the power function. The power spline function served to investigate the functional form, leading to the choice of the logistic-based specification. We did not succeed in estimation of the log-logistic decay function (11), as the estimate for the bending point approached zero. Other functional forms were not estimated because they only have a curvature in one direction.

Figure 4 shows the different characteristics of the four estimated decay functions, and can be compared with the point cloud in figure 2. The exponential decay function performs very poorly, which can be explained by the fact that the functional

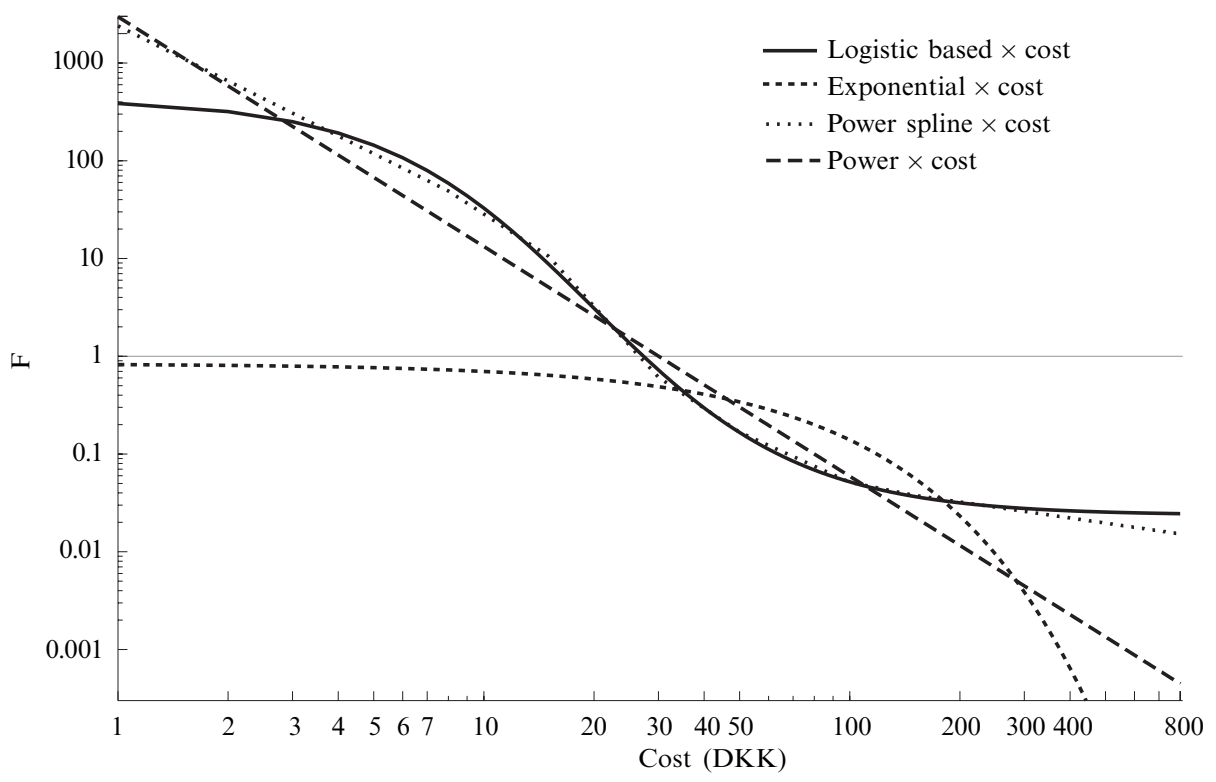

Figure 4. Various decay functions in a $\log -\log$ setting. 
form wrongly imposes strongly increasing elasticities. The exponential decay function can be useful for short distances, but here it is estimated over a wide range of distances. The incorrect functional form also causes implausible estimates for dummy parameters. The logistic-based and the power spline decay function are close to each other, while the power decay function is the best possible linear approximation. For the logistic-based decay function the standard error of the residuals is slightly higher than for the power spline function. However, the logistic-based specification uses four parameters fewer, and does not depend on a choice of kink points. We conclude that $\log$ trips can be adequately described as a logistic function of log travel cost, in combination with balancing factors for origins and destinations.

\section{Evaluation}

In this paper we have analyzed the form of the distance-decay function for commuting between the 275 municipalities in Denmark in 1995. We estimated the distance-decay function by NLWLS in logs. The balancing factors were estimated as dummy parameters, as Cesario (1974) did, but with a different computational method. To correct for the bias caused by taking logs, we used the log of flows plus $1 / 2$ (Sen and Soot, 1981). Most of the commuting flows are small and there are many zero flows. To correct for heteroscedasticity we used weights, based on a combination of specification error and Poisson error. This specification of weights, which requires iteration, is, to our knowledge, new. We also used the weights in the estimation of the balancing factors. We investigated various functional forms and finally chose a logistic-based form for the distance-decay function.

Commuting between municipalities in Denmark cannot be described by an exponential distance-decay function, while a power distance-decay function satisfies only on an intermediate cost range. But a specification where the logarithm of the decay function is a downward logistic function of $\log$ travel cost fits the data well. The distance-decay function we found could be used as a building block in the commuting submodel of a spatial general equilibrium model for Denmark, such as the LINE model (Madsen and Jensen-Butler, 2004). The elasticity of -2.35 estimated in the power specification gives a too simple representation of reality. For the logistic-based decay function, the elasticity with respect to travel cost is (in absolute value) small for small distances, reaches a value of 3.7 for distances around $20 \mathrm{~km}$, and approaches zero for large distances. Table 3 lists the values of the decay functions for various values of costs (which can roughly be interpreted as kilometers), and table 4 the elasticities. It appears that on intermediate distances $(10$ to $40 \mathrm{~km})$, commuting is very sensitive for travel cost, while for long-distance commuting (over $100 \mathrm{~km}$ ) travel costs are unimportant.

Table 3. Value of decay functions for selected values of travel cost.

\begin{tabular}{rlccc}
\hline Cost & Exponential & Power & Power spline & Logistic based \\
\hline 0 & 0.84 & infinity & infinity & 429.06 \\
5 & 0.76 & 67.64 & 118.12 & 144.35 \\
10 & 0.70 & 13.26 & 28.46 & 32.76 \\
20 & 0.58 & 2.60 & 3.24 & 3.09 \\
25 & 0.53 & 1.54 & 1.29 & 1.37 \\
40 & 0.41 & 0.51 & 0.29 & 0.30 \\
60 & 0.29 & 0.20 & 0.12 & 0.11 \\
120 & 0.10 & 0.04 & 0.04 & 0.04 \\
400 & 0.00 & 0.00 & 0.02 & 0.03 \\
\hline
\end{tabular}


Table 4. Elasticity of decay functions with respect to travel cost, for selected values of travel cost.

\begin{tabular}{rllll}
\hline Cost & Exponential & Power & Power spline & Logistic based \\
\hline 0 & 0 & -2.35 & -1.87 & 0 \\
5 & -0.09 & -2.35 & -1.87 & -1.46 \\
10 & -0.18 & -2.35 & -2.44 & -2.86 \\
20 & -0.36 & -2.35 & -4.12 & -3.70 \\
25 & -0.45 & -2.35 & -4.12 & -3.59 \\
40 & -0.72 & -2.35 & -2.54 & -2.83 \\
60 & -1.08 & -2.35 & -1.70 & -1.97 \\
120 & -2.15 & -2.35 & -0.76 & -0.86 \\
400 & -7.17 & -2.35 & -0.54 & -0.16 \\
\hline
\end{tabular}

That the cost elasticity varies over distance is important for the evaluation of infrastructure projects. Major new links have been constructed in Denmark, such as the Great Belt crossing. The opening of roads or railways will reduce generalized transport cost, stimulate commuting, and affect regional housing and labor markets. Knowledge of the distance-decay function is important to predict the size of these effects. In this paper we estimated only a single equation, not a complete model, and that is insufficient to make predictions for the commuting flows. However, in the completely specified models mentioned in section 2 , the effect of travel costs on other variables, like balancing factors and inflow and outflow, is only through the distancedecay function, so it is useful to compare the elasticities of different decay functions. The use of a power-decay function, which imposes constant elasticity, might lead to overestimation of the effect of new long-distance connections, and underestimation of the effect of regional infrastructure improvements.

In appendix B we derive several properties of the logistic-based decay function (25). For short distances the logistic-based decay function can be approached by an exponential decay function. For intermediate distances it can be approached by a power decay function. This is in accordance with the observation of Fotheringham and O'Kelly (1989) cited in section 1. The logistic-based decay function can unify these approaches in a single model. The various functional forms found in empirical studies can be interpreted as approximations of certain parts of an S-shaped curve, and the estimated elasticity in a power specification will depend on the cost range in the data. For long distances the elasticity in the logistic-based decay function approaches zero. The commonly used functional forms for distance decay do not allow that. The treatment of heteroscedasticity affects the weight of various parts of the decay function, as low costs are associated with large flows and high costs with small flows. In the absence of weighting, the many small flows, especially zero flows, will dominate the result. If the weights are based on a Poisson distribution, a few large flows on short distance will dominate. To avoid such problems, we took into account both specification error and the Poisson process.

We do not want to state that a logistic-based decay function is the ultimate truth. We do, however, clearly find an S-shaped function, and our logistic-based function fits the data well. Of course, this only refers to one dataset for one country, so we cannot make a general claim. However, this is supported by similar findings in the literature: a log-logistic decay function (Geurs and Ritsema van Eck, 2003; Hilbers and Verroen, 1993) or other S-shaped decay function (Johansson et al, 2002; 2003) and the theoretical work of Thorsen et al (1999). It is an open question for further research how this functional form can be explained from utility maximizing behavior. Willigers (2006, page 91) relates the flattening right tail of the decay function to the observation that 
the multinomial logit model systematically tends to underestimate the probability that a very unattractive alternative is chosen. We have demonstrated that allowing a more flexible form for the distance-decay function greatly improves the result in one application, so this approach should at least be considered in other applications.

Acknowledgements. The authors would like to thank Anne Kaag Andersen for making the data available and related help. We thank Jos van Ommeren and three anonymous referees for constructive comments on earlier versions.

\section{References}

Alonso W, 1978, “A theory of movements", in Human Settlement Systems: International Perspectives on Structure, Change and Public Policy Ed. N M Hansen (Ballinger, Cambridge, MA) pp 197-211

Andersen A K, 1999a Location and Commuting PhD thesis, Institute of Economics, University of Copenhagen

Andersen A K, 1999b, "Modelling commuting and residential location-spatial interaction models", in Location and Commuting PhD thesis, Institute of Economics, University of Copenhagen

Batten D F, Boyce D E, 1986, "Spatial interaction, transportation, and interregional commodity flow models", in Handbook of Regional and Urban Economics Ed. P Nijkamp (North-Holland, Amsterdam) pp $357-406$

Batty M, Sikdar P K, 1982, "Spatial aggregation in gravity models. 1. An information-theoretic framework" Environment and Planning A 14377 - 405

Bikker J A, 1987, "An international trade flow model with substitution: an extension of the gravity model” Kyklos $\mathbf{4 0} 315$ - 337

Bikker J A, de Vos A F, 1992, "A regional supply and demand model for inpatient hospital care" Environment and Planning A $\mathbf{2 4} 1097-1116$

Box G E P, Cox D R, 1964, “An analysis of transformations” Journal of the Royal Statistical Society. Series B (Methodological) $26211-252$

Carey H C, 1858 Principles of Social Science (Lippincott, Philadelphia, PA)

Cesario F J, 1974, "The interpretation and calculation of gravity model zone-to-zone adjustment factors" Environment and Planning A $6247-257$

Choukroun J-M, 1975, "A general framework for the development of gravity-type trip distribution models" Regional Science and Urban Economics 5 177-202

Cochrane R A, 1975, "A possible economic basis for the gravity model” Journal of Transport Economics and Policy $934-49$

de Vries J J, Nijkamp P, Rietveld P, 2001, “Alonso's theory of movements: developments in spatial interaction modeling" Journal of Geographical Systems $3233-256$

de Vries J J, Nijkamp P, Rietveld P, 2002, "Estimation of Alonso's theory of movements by means of instrumental variables" Networks and Spatial Economics 2(2) $107-126$

Doornik J A, 1998 Ox, An Object-oriented Matrix Programming Language Timberlake Consultants, London

Fik T J, Mulligan G F, 1998, "Functional form and spatial interaction models" Environment and Planning A $301497-1507$

Fotheringham A S, 1983, "A new set of spatial-interaction models: the theory of competing destinations" Environment and Planning A $1515-36$

Fotheringham A S, 1984, "Spatial flows and spatial patterns" Environment and Planning A 16 $529-543$

Fotheringham A S, O'Kelly M E, 1989 Spatial Interaction Models: Formulations and Applications (Kluwer, Dordrecht)

Geurs K T, Ritsema van Eck J R, 2003, "Evaluation of accessibility impacts of land-use scenarios: the implications of job competition, land-use, and infrastructure developments for the Netherlands" Environment and Planning B: Planning and Design 3069 -87

Hamerslag R, 1980, "Spatial development, developments in traffic and transportation, and changes in the transportation system", in Changes in the Field of Transport Studies Eds J B Polak, J B van der Kamp (Martinus Nijhoff, The Hague) pp 60-80

Hilbers H D, Verroen E J, 1993, "Het beoordelen van de bereikbaarheid van lokaties" [The evaluation of the accessibility of locations], report INRO-VVG 1993-09, TNO, Delft

Johansson B, Klaesson J, Olsson M, 2002, "Time distances and labor market integration" Papers in Regional Science $\mathbf{8 1} 305-327$

Johansson B, Klaesson J, Olsson M, 2003, "Commuters' non-linear response to time distances" Journal of Geographical Systems 5 315-329 
Ledent J, 1980, “Calibrating Alonso's general theory of movement: the case of interprovincial migration flows in Canada" Sistemi Urbani 2327 - 358

Madsen B, Jensen-Butler C, 2004, "Theoretical and operational issues in sub-regional economic modelling, illustrated through the development and application of the LINE model" Economic Modelling $21471-508$

Mazurkiewicz L, 1982, "A spatial interaction model with the distance decay function based on a random variable distribution" Environment and Planning A 14789 - 793

Nijkamp P, Reggiani A, 1992 Interaction, Evolution and Chaos in Space (Springer, Berlin)

Nijkamp P, Rouwendal J (Eds), 2004, "Special issue on advances in commuting studies" Growth and Change 35(3)

Pellegrini P A, Fotheringham A S, 1999, "Intermetropolitan migration and hierarchical destination choice: a disaggregate analysis from the US Public Use Microdata Samples" Environment and Planning A $311093-1118$

Poot J, 1986, "A system approach to modelling the inter-urban exchange of workers in New Zealand" Scottish Journal of Political Economy $33249-274$

Rouwendal J, Nijkamp P, 2004, "Living in two worlds: a review of home-to-work decisions" Growth and Change $35287-303$

Sen A, Smith T E, 1995 Gravity Models of Spatial Interaction Behavior (Springer, Berlin)

Sen A, Soot S, 1981, "Selected procedures for calibrating the generalized gravity model" Papers of the Regional Science Association 48165 - 176

Stewart J Q, 1941, “An inverse distance variation for certain social influences” Science 93 89-90

Stoer J, Bulirsch R, 1980 Introduction to Numerical Analysis (Springer, New York)

Taylor P J, 1975 Distance Decay Models in Spatial Interactions (Geo Abstracts, Norwich, Norfolk)

Thorsen I, Uboe J, Naevdal G, 1999, “A network approach to commuting” Journal of Regional Science $3973-101$

Tiefelsdorf M, 2003, "Misspecifications in interaction model distance decay relations: a spatial structure effect" Journal of Geographical Systems 5(1) 25-50

Willigers J, 2006 Impact of High-speed Railway Accessibility on the Location Choices of Office Establishments PhD thesis, Faculty of Geosciences, Universiteit Utrecht

Wilson A G, 1971, "A family of spatial interaction models, and associated developments" Environment and Planning $31-32$ 


\section{Appendix A}

\section{Weighted least-squares with balancing factors}

In this appendix we present the formulas for the inclusion of balancing factors in the weighted least-squares (WLS) estimation method that was described in section 4. To simplify the notation we use lowercase for logs of variables, where $t_{i j}$ stands for $\ln \left(T_{i j}+\frac{1}{2}\right)$ and $f_{i j}$ for $\mathrm{f}\left(G_{i j}\right)$. We can then write equation (13) as

$$
t_{i j}-o_{i}-d_{j}+q=a_{i}+b_{j}+f_{i j}+u_{i j},
$$

where $f_{i j}$ is a submodel which will generally contain multiple explanatory variables, and parameters $\gamma$. Usually, $f_{i j}$ is nonlinear, but, as each step of the nonlinear WLS iteration uses a linear approximation, we can assume linearity here.

We want to minimize the weighted sum of squared residuals

$$
\sum_{i j} w_{i j}^{2} \hat{u}_{i j}^{2} \text {. }
$$

There are three groups of parameters: $a_{i}$ are parameters of origin dummies, $b_{j}$ are parameters of destination dummies, and $\gamma$ is the vector of parameters in $f$ (including a constant). Both sets of balancing factors are identified only up to a constant. Therefore, we impose that weighted sums of $a_{i}$ and of $b_{j}$ are zero:

$$
\begin{aligned}
& \sum_{i} w_{i}^{(a)} \hat{a}_{i}=0, \\
& \sum_{j} w_{j}^{(b)} \hat{b}_{j}=0 .
\end{aligned}
$$

These weights are chosen in a way that is convenient in the estimation procedure:

$$
\begin{aligned}
& w_{i}^{(a)}=\left(\sum_{j} w_{i j}^{2}\right)^{\frac{1}{2}}, \\
& w_{j}^{(b)}=\left(\sum_{i} w_{i j}^{2}\right)^{\frac{1}{2}} .
\end{aligned}
$$

We include the identification restrictions (A3) and (A4) as artificial observations in the estimation procedure.

Generally, the parameter estimates for WLS are given by the following formula:

$$
\left(\mathbf{X}^{\mathrm{T}} \mathbf{W X}\right)^{-1} \mathbf{X}^{\mathrm{T}} \mathbf{W} \boldsymbol{y} \text {. }
$$

In this case $\mathbf{X}$ consists of three groups of columns: $k$ vectorized cost variables (derivatives of $f$ with respect to the parameters), $n$ origin dummies, and $m$ destination dummies:

$$
\mathbf{X}=\left[\begin{array}{ccc}
\frac{\mathrm{d}[\operatorname{vec}(f)]}{\mathrm{d} \gamma^{\mathrm{T}}} & \mathbf{I}_{n} \otimes \boldsymbol{I}_{m} & \boldsymbol{1}_{n} \otimes \mathbf{I}_{m} \\
\boldsymbol{0} & \left\{w_{i}^{(a)}\right\}_{1 \times n} & \boldsymbol{0} \\
\boldsymbol{0} & \boldsymbol{0} & \left\{w_{j}^{(b)}\right\}_{1 \times m}
\end{array}\right] .
$$

The first row of expressions represents $n m$ rows of $\mathbf{X}$, the other two rows each a single row, representing the identification restrictions. The Kronecker product is used to represent the pattern of zeros and ones of the origin and destination dummies. 
The notation $\left\{a_{i j}\right\}_{n \times m}$ represent an $n \times m$ matrix with typical element $a_{i j}$ ( $i$ always corresponds to $n, j$ to $m$, and the elements of $\gamma$ to $k$ ). $\mathbf{W}$ is a diagonal matrix of size $n m+2$, with the $w_{i j}^{2}$ as elements (followed by two ones for the identification restrictions).

$$
\mathbf{W}=\left[\begin{array}{cc}
\operatorname{diag}\left[\operatorname{vec}\left(\left\{w_{i j}^{2}\right\}_{n \times m}\right)\right] & \boldsymbol{0} \\
\boldsymbol{0} & \mathbf{I}_{2}
\end{array}\right] .
$$

We write $y$ for the left-hand side of equation (A1), or its equivalent in the nonlinear procedure. Then

$$
\mathbf{X}^{\mathrm{T}} \mathbf{W} \boldsymbol{y}=\left[\begin{array}{c}
\frac{\mathrm{d}\left[\operatorname{vec}(f)^{\mathrm{T}}\right]}{\mathrm{d} \gamma} \cdot \operatorname{vec}\left(\left\{w_{i j}^{2} y_{i j}\right\}_{n \times m}\right) \\
\left\{\sum_{j} w_{i j}^{2} y_{i j}\right\}_{n \times 1} \\
\left\{\sum_{i} w_{i j}^{2} y_{i j}\right\}_{m \times 1}
\end{array}\right] .
$$

$\mathbf{X}^{\mathrm{T}} \mathbf{W X}$ is a symmetric matrix of size $k+n+m$. We partition it into nine blocks (subscript $c$ corresponds to $\gamma$, etc):

$$
\mathbf{X}^{\mathrm{T}} \mathbf{W X}=\left[\begin{array}{lll}
\left(\mathbf{X}^{\mathrm{T}} \mathbf{W X}\right)_{c c} & \left(\mathbf{X}^{\mathrm{T}} \mathbf{W X}\right)_{c a} & \left(\mathbf{X}^{\mathrm{T}} \mathbf{W X}\right)_{c b} \\
\left(\mathbf{X}^{\mathrm{T}} \mathbf{W X}\right)_{a c} & \left(\mathbf{X}^{\mathrm{T}} \mathbf{W X}\right)_{a a} & \left(\mathbf{X}^{\mathrm{T}} \mathbf{W X}\right)_{a b} \\
\left(\mathbf{X}^{\mathrm{T}} \mathbf{W X}\right)_{b c} & \left(\mathbf{X}^{\mathrm{T}} \mathbf{W X}\right)_{b a} & \left(\mathbf{X}^{\mathrm{T}} \mathbf{W X}\right)_{b b}
\end{array}\right] .
$$

Then the blocks are as follows:

$$
\begin{aligned}
& \left(\mathbf{X}^{\mathrm{T}} \mathbf{W} \mathbf{X}\right)_{c c}=\frac{\mathrm{d}\left[\operatorname{vec}(f)^{\mathrm{T}}\right]}{\mathrm{d} \gamma} \cdot \operatorname{diag}\left[\operatorname{vec}\left(\left\{w_{i j}^{2}\right\}_{n \times m}\right)\right] \cdot \frac{\mathrm{d}[\operatorname{vec}(f)]}{\mathrm{d} \gamma^{\mathrm{T}}}, \\
& \left(\mathbf{X}^{\mathrm{T}} \mathbf{W} \mathbf{X}\right)_{a c}=\left(\mathbf{X}^{\mathrm{T}} \mathbf{W} \mathbf{X}\right)_{c a}^{\mathrm{T}}=\left\{\sum_{j} w_{i j}^{2} \frac{\mathrm{d} f_{i j}}{\left.\mathrm{~d} \gamma^{\mathrm{T}}\right\}_{n \times k},}\right. \\
& \left(\mathbf{X}^{\mathrm{T}} \mathbf{W} \mathbf{X}\right)_{b c}=\left(\mathbf{X}^{\mathrm{T}} \mathbf{W} \mathbf{X}\right)_{c b}^{\mathrm{T}}=\left\{\sum_{i} w_{i j}^{2} \frac{\mathrm{d} f_{i j}}{\mathrm{~d} \gamma^{\mathrm{T}}}\right\}_{m \times k}, \\
& \left(\mathbf{X}^{\mathrm{T}} \mathbf{W} \mathbf{X}\right)_{a a}=\operatorname{diag}\left(\left\{w_{i}^{(a)}\right\}_{n}\right) \cdot\left(\mathbf{I}_{n}+\boldsymbol{1}_{n} \boldsymbol{1}_{n}^{\mathrm{T}}\right) \cdot \operatorname{diag}\left(\left\{w_{i}^{(a)}\right\}_{n}\right), \\
& \left(\mathbf{X}^{\mathrm{T}} \mathbf{W X}\right)_{b b}=\operatorname{diag}\left(\left\{w_{j}^{(b)}\right\}_{m}\right) \cdot\left(\mathbf{I}_{m}+\boldsymbol{1}_{m} \boldsymbol{1}_{m}^{\mathrm{T}}\right) \cdot \operatorname{diag}\left(\left\{w_{j}^{(b)}\right\}_{m}\right), \\
& \left(\mathbf{X}^{\mathrm{T}} \mathbf{W} \mathbf{X}\right)_{a b}=\left(\mathbf{X}^{\mathrm{T}} \mathbf{W} \mathbf{X}\right)_{b a}^{\mathrm{T}}=\left\{w_{i j}^{2}\right\}_{n \times m} \cdot
\end{aligned}
$$

It is possible to invert $\left(\mathbf{X}^{\mathrm{T}} \mathbf{W X}\right)_{a a}$ and $\left(\mathbf{X}^{\mathrm{T}} \mathbf{W} \mathbf{X}\right)_{b b}$ analytically. However, that does not hold for the combined block

$$
\left[\begin{array}{ll}
\left(\mathbf{X}^{\mathrm{T}} \mathbf{W X}\right)_{a a} & \left(\mathbf{X}^{\mathrm{T}} \mathbf{W X}\right)_{a b} \\
\left(\mathbf{X}^{\mathrm{T}} \mathbf{W X}\right)_{b a} & \left(\mathbf{X}^{\mathrm{T}} \mathbf{W X}\right)_{b b}
\end{array}\right] .
$$

This makes it impossible to implement WLS with balancing factors using a decomposition as proposed by Cesario (1974, page 252) and described by Sen and Smith (1995, page 477). The use of weighted averages in the decomposition (de Vries et al, 2002) does not solve this problem. As can be seen from the formulas above, weighted sums play an important role in the estimation method. However, an analytical solution is hampered by the interaction of the balancing factors through all the different weights in $\left(\mathbf{X}^{\mathrm{T}} \mathbf{W} \mathbf{X}\right)_{a b}$. 


\section{Appendix B}

\section{The logistic function}

The logistic-based distance-decay function,

$$
\mathrm{f}\left(G_{i j}\right)=\gamma_{0}+\gamma_{1}\left[1+\left(\frac{G_{i j}}{\gamma_{2}}\right)^{\gamma_{3}}\right]^{-1},
$$

has some interesting properties. If cost approaches zero, $G_{i j} \downarrow 0$, then

$$
\mathrm{f}\left(G_{i j}\right) \approx \gamma_{0}+\gamma_{1}\left[1-\left(\frac{G_{i j}}{\gamma_{2}}\right)^{\gamma_{3}}\right]=\gamma_{0}+\gamma_{1}-\gamma_{1}\left(\frac{G_{i j}}{\gamma_{2}}\right)^{\gamma_{3}} .
$$

Now if the steepness parameter $\gamma_{3}=1$, we have

$$
\mathrm{f}\left(G_{i j}\right) \approx \gamma_{0}+\gamma_{1}-\frac{\gamma_{1}}{\gamma_{2}} G_{i j}
$$

This is an exponential distance-decay function [compare equation (21), with a parameter transformation]. So, if the steepness parameter equals 1, the logistic-based decay function approaches an exponential decay function for low cost. Around the bending point the logistic function can be approximated by a linear function (in $\ln G_{i j}$ ), so for intermediate distances the logistic-based function can be approximated by a power decay function. If cost becomes very large, $G_{i j} \rightarrow \infty$, then the distance-decay function approaches an inverse exponential function:

$$
\mathrm{f}\left(G_{i j}\right) \approx \gamma_{0}+\gamma_{1}\left(\frac{G_{i j}}{\gamma_{2}}\right)^{-\gamma_{3}}
$$

and the elasticity

$$
\frac{\mathrm{df}\left(G_{i j}\right)}{\mathrm{d} \ln G_{i j}} \approx-\gamma_{3} \gamma_{1}\left(\frac{G_{i j}}{\gamma_{2}}\right)^{-\gamma_{3}} .
$$

So, if cost rises by a certain factor, the elasticity decreases by a given power of that factor. This is the property that we noted in section 5 in the estimated elasticities for the power spline function: the slope halves for equal increases in $\ln G_{i j}$.

The elasticity of trips with respect to cost is

$$
\frac{\operatorname{df}\left(G_{i j}\right)}{\operatorname{d} \ln G_{i j}}=-\gamma_{1} \gamma_{3}\left[1+\left(\frac{G_{i j}}{\gamma_{2}}\right)^{\gamma_{3}}\right]^{-1}\left\{1-\left[1+\left(\frac{G_{i j}}{\gamma_{2}}\right)^{\gamma_{3}}\right]^{-1}\right\} \text {. }
$$

At $G_{i j}=0$, the elasticity equals zero. The largest elasticity (in absolute value) is attained for $G_{i j}=\gamma_{2}$, and equals $-\frac{1}{4} \gamma_{1} \gamma_{3}$. For $G_{i j} \rightarrow \infty$, the elasticity approaches zero. 
Conditions of use. This article may be downloaded from the E\&P website for personal research by members of subscribing organisations. This PDF may not be placed on any website (or other online distribution system) without permission of the publisher. 